\title{
THE GEOMETRY OF FRACTIONAL STABLE MATCHINGS AND ITS APPLICATIONS
}

\author{
CHUNG-PIAW TEO AND JAY SETHURAMAN
}

\begin{abstract}
We study the classical stable marriage and stable roommates problems using a polyhedral approach. We propose a new LP formulation for the stable roommates problem, which has a feasible solution if and only if the underlying roommates problem has a stable matching. Furthermore, for certain special weight functions on the edges, we construct a 2-approximation algorithm for the optimal stable roommates problem. Our technique exploits features of the geometry of fractional solutions of this formulation. For the stable marriage problem, we show that a related geometry allows us to express any fractional solution in the stable marriage polytope as a convex combination of stable marriage solutions. This also leads to a genuinely simple proof of the integrality of the stable marriage polytope.
\end{abstract}

1. Introduction. In this paper we use a polyhedral approach to study stable matching problems with strict preferences. The two versions of the stable matching problem we study are the stable marriage problem and the stable roommates problem. In the marriage problem, there are two finite, disjoint sets of men and women. Each man has preferences over the women, and each woman has preferences over the men. For simplicity of presentation, we assume that the number of men and women are identical, and that each individual's preferences are strict. A matching $M$ of the men to the women is unstable if there is a man $m$ and woman $w$ such that $m$ and $w$ prefer each other to their assigned partners under $M$; in this case, $(m, w)$ is called a blocking pair. In their pioneering paper, Gale and Shapley (1962) showed that every instance of the stable marriage problem has a stable matching. The structure of the marriage problem has been extensively studied in the subsequent literature. The computer science community has extended and improved on Gale and Shapley's algorithm, identifying many new structural properties of the marriage problem. (See for instance Knuth 1976, Gusfield and Irving 1989.) Interest within the economics community was aroused by Roth's discovery (Roth 1984) that a version of the stable marriage algorithm, which evolved by a trial-and-error process that spanned more than half a century, has been in use since 1951 in the Hospital-Residents market. (Roth and Sotomayor 1991 contains further details of the use of the marriage problem as a model for two-sided matching markets.) Vande Vate (1989) initiated the study of the marriage problem using a mathematical programming approach. He obtained a complete characterization of the convex hull of the stable marriage solutions. This polyhedral description has been extended by Rothblum (1992) to the case when the matching need not be perfect (i.e., some men would rather remain single than be matched to certain women and vice versa). Since the original proof by Vande Vate is rather complicated, several simpler proofs have been proposed in the literature (Rothblum 1992, Roth, Rothblum and Vande Vate 1993).

A related question raised by the Gale-Shapley algorithm is the issue of fairness in the stable marriage problem. It is well known that the Gale-Shapley algorithm returns either a men-optimal or a women-optimal stable marriage solution, depending on the implementation. For the men-optimal solution obtained by the algorithm, each man is matched

Received May 6, 1996; revised March 21, 1997, October 21, 1997, March 9, 1998 and May 11, 1998. AMS 1991 subject classification. Primary: 90C27; Secondary: 05C70, 68Q25.

OR/MS subject classification. Primary: Programming/Linear, Integer; Secondary: Analysis of algorithms/Approximation.

Key words. Stable matching, linear programming, rounding, approximation algorithms. 
to the best partner he can hope for under the stability condition, which (curiously) also results in each woman being matched to her worst possible partner in any stable marriage. (Not surprisingly, an obvious analogous result holds for the women-optimal solution.) Several studies have been reported on finding stable marriage solutions that are "fair" (in some sense) to both sexes. Irving, Leather and Gusfield (1987) introduced and studied the egalitarian stable marriage problem: They showed that a stable marriage solution that minimizes the objective function

$$
\sum_{m} \sum_{w}(m r(m, w)+w r(w, m))
$$

can be constructed in polynomial time. Here, $\operatorname{mr}(m, w)$ is the position of woman $w$ in man $m$ 's list, and $w r(w, m)$ is the position of man $m$ in woman $w$ 's list. Knuth (1976) described and solved (attributed to S. Selkow) the minimum-regret stable marriage problem. The regret of a marriage in this case is measured by the person (man or woman) who has the worst partner (in terms of ranking) in it. A minimum-regret stable marriage is a solution that attains the minimum regret among all the stable marriages.

In their pioneering work, Gale and Shapley also initiated the study of the stable roommates problem. In an instance of this problem, we have a set of $2 n$ persons (say college students) who need to be paired up as roommates. Each person has a preference list ranking all the other persons in the set. We assume all preferences are strict. A matching is unstable when two people are not paired in the matching, but prefer each other to their assigned roommates. Such a pair is called a blocking pair. A matching is stable if and only if there are no blocking pairs.

Unlike the stable marriage problem, the stable roommates problem need not have a solution. Knuth (1976), in one of his twelve unsolved problems associated with stable matching, asked whether the feasibility of an instance of the stable roommates problem can be settled in polynomial time. His question has been answered by Irving (1985), and also more recently by Subramanian (1994) who relates the problem to the more general network stability problem.

Feder (1992) showed that if weights of the type $c(i, j)+c(j, i)$ are imposed on the edge $\{i, j\}$, then the minimum cost version of the stable roommates problem is already NP-hard, even if the cost functions $\{c(i, j)\}$ are restricted to the class which satisfies:

$$
c(i, j) \leq c(i, k) \quad \text { only if } i \text { prefers } j \text { to } k \text { for all } i, j, k .
$$

Gusfield and Irving (1989) designed a 2-approximation algorithm for this problem under this restriction.

Abeledo and Rothblum (1994) recently initiated the study of the polyhedral structure of the stable roommates problem. Generalizing Vande Vate's formulation for the marriage problem, they showed that many structural properties of the stable roommates problem can be derived via linear algebraic arguments, mainly via linear programming duality. However, the proposed formulation has a major weakness - it is not strong enough (to be used) to decide the existence of a stable matching. In other words, there are infeasible instances of the stable roommates problem for which the associated polytope is nonempty.

In this work, we continue the polyhedral study of the stable matching polytope. Our contributions are:

- Using an observation due to Roth, Rothblum and Vande Vate (1993), we show in §2 that any fractional solution in the stable marriage polytope can be written as a convex combination of stable marriage solutions in an elegant way, thus providing a genuinely simple proof of the integrality of the stable marriage polytope. (Abeledo, Blum and Rothblum 1996 have also recently obtained a more complicated approach to this problem.) We also prove an 
interesting structural property of the stable marriage solutions, generalizing the well-known median property first discovered by Conway (see Gusfield and Irving 1989). The proof technique uses a rounding approach, which captures the stability condition in a purely geometric way. More importantly, this paper proposes a new approach to address the asymmetry often observed in the stable marriage problem by showing the existence of a solution which is "fair" to everyone (men and women) involved in the problem.

- We propose an LP model, which can be used to handle objective functions of the type considered in the egalitarian version and/or the type considered in the minimumregret version of the stable marriage problem. This provides a unified and more general framework to discuss other issues of fairness in the marriage problem via LP methodology.

- We strengthen the fractional matching polytope (for the roommates case) studied in Abeledo and Rothblum (1994) by proposing a new class of valid inequalities for the roommates problem. Furthermore, we show that the proposed class of valid inequalities can be separated in polynomial time. We show further that the formulation studied in this paper can be used to settle the existence question of the stable roommates problem under strict preference lists, i.e., a stable roommates matching exists if and only if our polyhedron is nonempty. This provides a LP alternative to Irving's (Irving 1985) combinatorial algorithm for the decision version of the roommates problem. It also complements a recent study by Abeledo and Blum (1996) who showed that the feasibility question can be settled by solving a series of LP problems. For certain classes of cost functions on the edges, the stable roommates solution obtained from our formulation is within a factor of 2 of the optimum stable roommates solution.

It is also interesting to compare these results with the known results on perfect matching problems. Both the optimization version of the perfect matching problem and the feasibility version of the stable roommates problem are known to be solvable in polynomial time. Although the stable roommates problem can be addressed and understood by transforming the problems to the space of rotational posets (cf. Gusfield and Irving 1989), very little was known about the stable roommates polytope in the space of the natural edge variables prior to this work. The Odd Cyclic Preference inequalities which we have identified in this paper play an analogous role to the odd-set constraints in the perfect matching polytope. The latter, coupled with the matching constraints, gives rise to a complete characterization of the perfect matching polytope in nonbipartite graphs. The former, coupled with the matching and stability constraints, completely resolves the issue of feasibility of stable roommates problems. The proof technique used can be made constructive, giving rise to a LP-based heuristic to construct solutions for the optimal stable roommates problem. We refer the readers to Teo and Sethuraman (1997) for details of the experiments and some encouraging observations.

In the rest of this paper, we let $Z_{\mathrm{IP}}(\cdot)$ and $Z_{\mathrm{LP}}\left(^{\cdot}\right)$ denote respectively the optimal integral and fractional solutions to the underlying formulation of the stable matching problem. We will often omit presenting the IP formulations since these can be easily constructed from the LP formulations. Furthermore, we assumed that all participants in the problem instances must be matched in all stable matchings. In the marriage case, most of the results considered here are directly applicable to the more general version with nonperfect matching because of Roth's result that the same participants are matched in every stable matching (Roth 1984).

2. Stable marriage polytope revisited. For women $w_{i}$ and $w_{j}$, we write $w_{i}>_{m} w_{j}$ if man $m$ prefers $w_{i}$ to $w_{j}$. Let

$$
x_{i, j}= \begin{cases}1 & \text { if } m_{i} \text { is matched to } w_{j} \\ 0 & \text { otherwise }\end{cases}
$$


Any incidence vector that corresponds to a stable matching is called a stable marriage solution. Consider the following relaxation of the problem (Vande Vate 1989):

$$
\begin{gathered}
\sum_{j} x_{i, j}=1 \quad \forall i, \\
\sum_{i} x_{i, j}=1 \quad \forall j, \\
x_{i, j}+\sum_{k: w_{k}<m_{i} w_{j}} x_{i, k}+\sum_{k: m_{k}<w_{j} m_{i}} x_{k, j} \leq 1 \quad \forall i, j, \\
x_{i, j} \geq 0 \quad \forall i, j .
\end{gathered}
$$

Inequality (3) is clearly valid, otherwise, we must have $\sum_{k: m_{k}<{ }_{w} m_{i}} x_{k, j}=1$ and $\Sigma_{k: w_{k}<m_{i} w_{j}} x_{i, k}$ $=1$. So $m_{i}$ and $w_{j}$ are matched to less favourable mates in the matching and thus $\left(m_{i}\right.$, $w_{j}$ ) is a blocking pair. Note that the polytope $\left(P_{S M}\right)$ is always nonempty (cf. Gale and Shapley 1962).

Theorem 1 (VANDE VATE 1989). The polytope $\left(P_{S M}\right)$ is the convex hull of the stable marriage solutions.

To give a simple proof of this result, we need the following known property of the stable marriage polytope:

Lemma 1 (Roth, Rothblum and VANDe VAte 1993). Let $x$ be a feasible solution in the stable marriage polytope. Then

$$
x_{i, j}>0 \quad \text { implies } x_{i, j}+\sum_{k: w_{k}<m_{i} w_{j}} x_{i, k}+\sum_{k: m_{k}<w_{j} m_{i}} x_{k, j}=1 \text {. }
$$

For the sake of completeness, we sketch the proof from Roth, Rothblum and Vande Vate (1993).

Proof. Consider the primal problem

$$
\min \left\{\sum_{i, j} x_{i, j}: x \in\left(P_{S M}\right)\right\}
$$

and its dual

$$
\begin{aligned}
& \max \sum_{i} \alpha_{i}+\sum_{j} \beta_{j}-\sum_{i, j} \gamma_{i, j} \\
& \text { subject to } \alpha_{i}+\beta_{j}-\sum_{k: w_{k} \geq_{m_{i}} w_{j}} \gamma_{i, k}-\sum_{k: m_{k}>w_{j} m_{i}} \gamma_{k, j} \leq 1, \quad \forall i, j, \\
& \gamma_{i, j} \geq 0, \quad \forall i, j .
\end{aligned}
$$

Set $\alpha_{i}=\Sigma_{j} \gamma_{i, j}, \beta_{j}=\Sigma_{i} \gamma_{i, j}, \gamma_{i, j}=x_{i, j}$. Then the constraints in the dual reduce to

$$
\gamma_{i, j}+\sum_{k: w_{k}<m_{i} w_{j}} \gamma_{i, k}+\sum_{k: m_{k}<w_{j} m_{i}} \gamma_{k, j} \leq 1, \quad \forall i, j
$$


which is clearly true if $\gamma_{i, j}=x_{i, j}$ and $x$ is feasible in the primal. Furthermore, this is a dual solution with objective function value $\sum_{i, j} x_{i, j}$. Hence the lemma follows directly from the complementary slackness property of optimal primal and dual solutions.

We show next that Lemma 1 gives rise to a particularly interesting geometry for the stable marriage problem. Note that since $\Sigma_{j} x_{i, j}=1$, then for each man $m_{i}$, we can construct $n$ intervals of the type ( $a, b]$ (left-open, right-closed) of length $x_{i, j}$-one for each woman $w_{j}$ (some of these intervals can be empty). We can arrange these $n$ intervals in any nonoverlapping order to cover the line segment $(0,1]$. We can do the same for the women. By slight abuse of terminology, we will also denote by $x_{i, j}$ 's the $n$ disjoint subintervals in $(0,1]$ that correspond to the arrangement.

Proof of Theorem 1. Let $x$ be any feasible solution in $P_{S M}$. We show that $x$ can be written as a convex combination of stable matchings. Consider the following table of $2 n$ rows: For each man $m_{i}, x_{i, j}, j=1, \ldots, n$ are arranged in decreasing preference of $m_{i}$ to cover the interval $(0,1]$. For each woman $w_{j}, x_{i, j}, i=1, \ldots, n$ are arranged in increasing preference of $w_{j}$ to cover the interval $(0,1]$. Note that Lemma 1 suggests a nice property of the arrangement, i.e.,

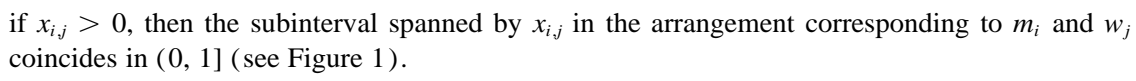

We generate a random number $U$ uniformly in $(0,1]$ and construct a matching in the following way:

Match $m_{i}$ to $w_{k}$ if $x_{i, k}>0$ and in the row corresponding to $m_{i}, U$ lies in the sub-interval spanned by $x_{i, k}$ in $(0,1]$. Similarly, match $w_{j}$ to $m_{l}$ if in the row corresponding to $w_{j}, U$ lies in the subinterval spanned by $x_{l, j}$ in $(0,1]$.

By Lemma 1, and by the way we construct the table (see Figure 1), $m_{i}$ is matched to $w_{j}$ if and only if $w_{j}$ is matched to $m_{i}$. Furthermore, no two men can be matched to the same woman, and similarly no two women can be matched to the same man. So the above assignment gives rise to a perfect matching. Any woman ( say $w_{k}$ ) who is preferred by $m_{i}$ to his mate $w_{j}$ in this assignment (i.e., the subinterval $x_{i, k}$ is on the left of $x_{i, j}$ in the arrangement by $m_{i}$ ) is assigned a mate whom she strictly prefers to $m_{i}$, since in the row

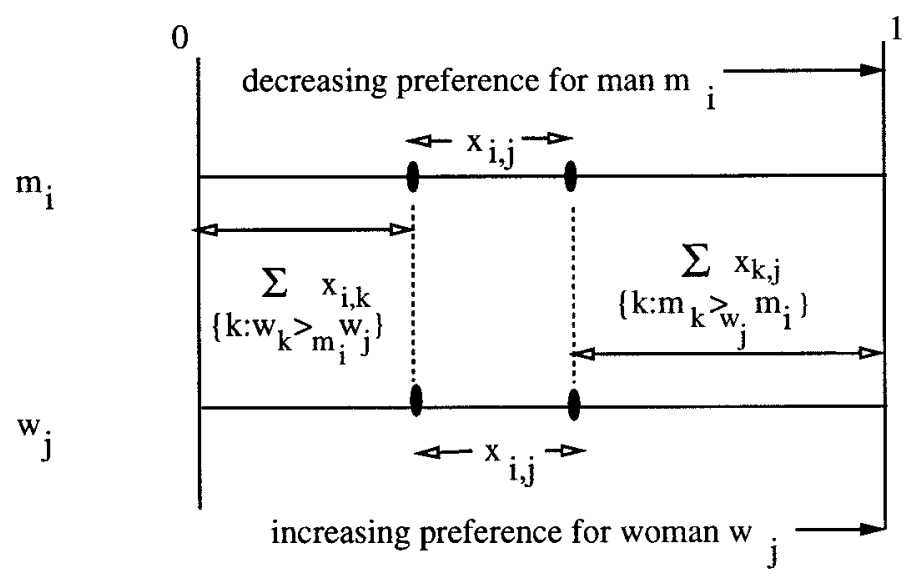

FIGURE 1. The table of arrangements. 
(in the table) corresponding to $w_{k}$, the random number $U$ lies strictly to the right of the subinterval $x_{i, k}$. Hence the matching is stable.

Let $X_{U}$ be the assignment obtained. In other words, $X_{U}(i, j)=1$ if and only if $m_{i}$ and $w_{j}$ are matched to each other under the above assignment. Then

$$
E\left(X_{U}(i, j)\right)=P\left(U \text { lies in the subinterval spanned by } x_{i, j}\right)=x_{i, j} \text {. }
$$

Thus $x(i, j)=\int_{0}^{1} X_{u}(i, j) d u$ and $x$ can be written as convex combination of $X_{u}$ as $u$ varies over the interval $(0,1]$. Note that there are at most $O\left(n^{2}\right)$ distinct assignments arising from $X_{u}$ as $u$ varies.

The above argument gives rise to an efficient constructive procedure to express any fractional stable marriage solution as a convex combination of integral stable marriage solutions:

- Let $x$ be a feasible fractional solution in the stable marriage polytope. [Using the values of $x$, construct the table of $2 n$ rows as in the proof of Theorem 1.]

- Sort the coordinates of the endpoints of all the subintervals. Each row gives rise to at most $n+1$ distinct coordinates, so there are at most $O\left(n^{2}\right)$ distinct coordinates obtained in this way, say they are $0=I_{1}<I_{2}<\cdots<I_{p}=1$, for some integer $p$.

- For each random number $U$ in the interval $\left(I_{k}, I_{k+1}\right]$, the assignment produced by $U$ in the randomized algorithm is identical. Let $X_{I_{k+1}}$ be the (integral) stable marriage solution obtained with $U=I_{k+1}$. Then

$$
x=\sum_{j=1}^{p-1}\left(I_{j+1}-I_{j}\right) X_{I_{j+1}} .
$$

The rounding approach can also be used to derive the following surprising property of the stable marriage solutions:

THEOREM 2. Let $X_{1}, X_{2}, \ldots, X_{l}$ be l distinct stable marriage solutions. Each man $m_{i}$ has $l$ possible mates under these matchings. Assign him the woman whose rank is $k$ among the $l$ (possibly nondistinct) women. For each woman $w_{j}$, assign her to the man whom she ranked $l+1-k$ among the $l$ men she was assigned to under the matchings. This assignment gives rise to another, not necessarily distinct, stable marriage solution.

Proof. Let $x(i, j)=(1 / l) \sum_{p=1}^{l} X_{p}(i, j)$. Then $x$ is a fractional point in the stable marriage polytope. Consider the case $U=k / l-\epsilon$ under the randomized rounding scheme, where $\epsilon>0$ is an arbitrarily small number. Every man is then assigned to the woman whose rank is $k$ among the $l$ matchings. Similarly, every woman is assigned to the man whose rank is $l+1-k$ among the $l$ matchings. Furthermore, it follows from the proof to Theorem 1 that the assignment is a stable marriage solution.

When $l=3$ and $k=2$, the above reduces to the well-known median property due to Conway (cf. Gusfield and Irving 1989). When $l$ corresponds to the total number of distinct stable marriage solutions in the problem, and $k=1$, the above gives rise to the men-optimal assignment considered in Gale and Shapley (1962). When the total number of solutions, $l$, is odd, the case $k=(l+1) / 2$ is of special interest - there is a stable marriage solution in which every person is assigned to a partner who is the "median" partner among all their possible mates! It is an interesting open problem to determine whether the median solution can be computed in polynomial time. 
ExAmPLE. Consider the following stable marriage instance (cf. Irving, Leather and Gusfield 1987).

\begin{tabular}{|c|c|c|c|}
\hline nan & decreasing & woman & decreasing prefe \\
\hline 1 & $\begin{array}{llllllll}3 & 1 & 5 & 7 & 4 & 2 & 8 & 6\end{array}$ & 1 & $\begin{array}{llllllll}4 & 3 & 8 & 1 & 2 & 5 & 7 & 6\end{array}$ \\
\hline 2 & 613348752 & 2 & $\begin{array}{llllllll}3 & 7 & 5 & 8 & 6 & 4 & 1 & 2\end{array}$ \\
\hline 3 & $\begin{array}{llllllll}7 & 4 & 3 & 6 & 5 & 1 & 2 & 8\end{array}$ & 3 & $\begin{array}{llllllll}7 & 5 & 8 & 3 & 6 & 2 & 1 & 4\end{array}$ \\
\hline 4 & $\begin{array}{lllllllll}5 & 3 & 8 & 2 & 6 & 1 & 4 & 7\end{array}$ & 4 & 642733158 \\
\hline 5 & $\begin{array}{llllllll}4 & 1 & 2 & 8 & 7 & 3 & 6 & 5\end{array}$ & 5 & $\begin{array}{llllllll}8 & 7 & 1 & 5 & 6 & 4 & 3 & 2\end{array}$ \\
\hline 6 & $\begin{array}{llllllll}6 & 2 & 5 & 7 & 8 & 4 & 3 & 1\end{array}$ & 6 & $\begin{array}{llllllll}5 & 4 & 7 & 6 & 2 & 8 & 3 & 1\end{array}$ \\
\hline 7 & $\begin{array}{llllllll}7 & 8 & 1 & 6 & 2 & 3 & 4 & 5\end{array}$ & 7 & $\begin{array}{llllllll}14 & 5 & 6 & 2 & 8 & 3 & 7\end{array}$ \\
\hline & 267718345 & 8 & $\begin{array}{lllllll}2 & 5 & 4 & 3 & 7 & 8 & 1\end{array}$ \\
\hline
\end{tabular}

We list below all 23 stable matchings given in terms of permutations. (For instance, in matching $M_{2}$, man 1 marries woman 1, man 2 marries woman 3, man 3 marries woman 7 , man 4 marries woman 5 etc.)

$$
\begin{array}{llllllllllllllll}
M_{1}=3 & 1 & 7 & 5 & 4 & 6 & 8 & 2 & M_{2}=1 & 3 & 7 & 5 & 4 & 6 & 8 & 2 \\
M_{3}=3 & 1 & 4 & 5 & 2 & 6 & 8 & 7 & M_{4}=3 & 1 & 7 & 8 & 4 & 5 & 6 & 2 \\
M_{5}=1 & 3 & 4 & 5 & 2 & 6 & 8 & 7 & M_{6}=1 & 3 & 7 & 8 & 4 & 5 & 6 & 2 \\
M_{7}=3 & 1 & 4 & 8 & 2 & 5 & 6 & 7 & M_{8}=1 & 3 & 4 & 8 & 2 & 5 & 6 & 7 \\
M_{9}=5 & 3 & 4 & 8 & 2 & 7 & 6 & 1 & M_{10}=1 & 4 & 3 & 5 & 2 & 6 & 8 & 7 \\
M_{11}=3 & 1 & 4 & 6 & 8 & 5 & 2 & 7 & M_{12}=5 & 4 & 3 & 8 & 2 & 7 & 6 & 1 \\
M_{13}=5 & 3 & 4 & 6 & 8 & 7 & 2 & 1 & M_{14}=1 & 4 & 3 & 6 & 8 & 5 & 2 & 7 \\
M_{15}=5 & 4 & 3 & 6 & 8 & 7 & 2 & 1 & M_{16}=5 & 4 & 1 & 8 & 2 & 7 & 6 & 3 \\
M_{17}=5 & 8 & 3 & 6 & 7 & 4 & 2 & 1 & M_{18}=5 & 8 & 1 & 6 & 7 & 4 & 2 & 3 \\
M_{19}=5 & 4 & 1 & 6 & 8 & 7 & 6 & 3 & M_{20}=7 & 8 & 1 & 6 & 3 & 4 & 2 & 5 \\
M_{21}=7 & 8 & 2 & 1 & 6 & 4 & 3 & 5 & M_{22}=1 & 4 & 3 & 8 & 2 & 5 & 6 & 7 \\
M_{23}=1 & 3 & 4 & 6 & 8 & 5 & 2 & 7 & & & & & & & &
\end{array}
$$

Now, we can arrange all 23 partners for each man according to his order of preference (with repetitions). That list is as follows: 


$$
\begin{aligned}
& \text { man } \\
& \begin{array}{cllllllllllllllllllllllll}
1 & : & 3 & 3 & 3 & 3 & 1 & 1 & 1 & 1 & 1 & 1 & 1 & 1 & 5 & 5 & 5 & 5 & 5 & 5 & 5 & 5 & 7 & 7 \\
2 & : 1 & 1 & 1 & 1 & 1 & 3 & 3 & 3 & 3 & 3 & 3 & 3 & 4 & 4 & 4 & 4 & 4 & 4 & 4 & 8 & 8 & 8 & 8 \\
3 & : 7 & 7 & 7 & 7 & 4 & 4 & 4 & 4 & 4 & 4 & 4 & 4 & 3 & 3 & 3 & 3 & 3 & 3 & 1 & 1 & 1 & 1 & 2 \\
4 & : 5 & 5 & 5 & 5 & 5 & 8 & 8 & 8 & 8 & 8 & 8 & 8 & 8 & 6 & 6 & 6 & 6 & 6 & 6 & 6 & 6 & 6 & 1 \\
5 & : 4 & 4 & 4 & 4 & 2 & 2 & 2 & 2 & 2 & 2 & 2 & 2 & 2 & 8 & 8 & 8 & 8 & 8 & 8 & 7 & 7 & 3 & 6 \\
6 & : & 6 & 6 & 6 & 6 & 6 & 5 & 5 & 5 & 5 & 5 & 5 & 5 & 5 & 7 & 7 & 7 & 7 & 7 & 7 & 4 & 4 & 4 & 4 \\
7 & : 8 & 8 & 8 & 8 & 8 & 6 & 6 & 6 & 6 & 6 & 6 & 6 & 6 & 6 & 2 & 2 & 2 & 2 & 2 & 2 & 2 & 2 & 3 \\
8 & : & 2 & 2 & 2 & 2 & 7 & 7 & 7 & 7 & 7 & 7 & 7 & 7 & 7 & 1 & 1 & 1 & 1 & 1 & 3 & 3 & 3 & 5 & 5
\end{array}
\end{aligned}
$$

In this arrangement, any column will yield a stable matching; the median stable matching is just the 12th column.

2.1. On finding "fair" stable marriage solutions. One approach to finding fair stable marriage solutions is to incorporate an appropriate objective function into the problem. Let $M(x), W(x)$ denote respectively the worst ranking obtained by a man and a woman under the stable marriage solution $x$. In this section, we propose an LP model to handle this problem when the objective function is of the type

$$
\sum_{i, j}(c(i, j)+c(j, i)) x_{i, j}+f(M(x))+g(W(x))
$$

where $(c(i, j)+c(j, i))$ is the weight on the edge $\{i, j\}$ and $f, g$ are nondecreasing functions of $M(x)$ and $W(x)$ respectively. This class of objective functions includes as special cases several versions of stable marriage problems that have been proposed in the literature to deal with the issues of fairness. For instance, the minimum regret stable marriage problem can be reduced to the above with $f(k)=g(k)=k^{2 n}$ for $k=1, \ldots$, $n$, and $c(i, j)=c(j, i)=0$ for all $i, j$.

Let $x$ be a stable marriage solution. For each man $m_{i}$, let $w_{i(1)}, w_{i(2)}, \ldots, w_{i(n)}$ be the ordering of the potential partners under his preferences. Similarly, we order the potential partners of $w_{j}$ by $m_{j(1)}, \ldots, m_{j(n)}$. We model the worst ranking obtained by a man and a woman under $x$ by

$$
M(x)=\sum_{k=1}^{n} y_{k}, \quad W(x)=\sum_{k=1}^{n} z_{k}
$$

where

$$
y_{k}=\max _{m_{i}}\left(\sum_{l \geq k} x_{i, i(l)}\right), \quad z_{k}=\max _{w_{j}}\left(\sum_{l \geq k} x_{j(l), j}\right), \quad k=1,2, \ldots, n .
$$

Note that in this way, $y_{k}=1$ only when some man has been assigned to a partner whom he ranked $k$ or worse in his preference list. Hence $\sum_{k=1}^{n} y_{k}$ and $\sum_{k=1}^{n} z_{k}$ model the worst ranking obtained by a man and a woman in $x$. 
Let $f(0)=g(0)=0$. A natural LP relaxation for the problem is as follows:

$$
\begin{gathered}
(M M) \quad \min \sum_{i, j}(c(i, j)+c(j, i)) x_{i, j}+\sum_{k=1}^{n}\left((f(k)-f(k-1)) y_{k}\right. \\
\left.+(g(k)-g(k-1)) z_{k}\right) \\
\text { subject to: } \quad \sum_{j} x_{i, j}=1 \quad \forall i,
\end{gathered}
$$

$$
\begin{gathered}
\sum_{i} x_{i, j}=1 \quad \forall j, \\
x_{i, j}+\sum_{k: w_{k}<m_{i} w_{j}} x_{i, k}+\sum_{k: m_{k}<w_{j} m_{i}} x_{k, j} \leq 1 \quad \forall i, j, \\
y_{k} \geq \sum_{l \geq k} x_{i, i(l)} \quad \forall i, k, \\
z_{k} \geq \sum_{l \geq k} x_{j(l), j} \quad \forall j, k, \\
x_{i, j}, y_{k}, z_{k} \geq 0, \quad \forall i, j, k .
\end{gathered}
$$

THEOREM 3. $Z_{L P}(M M)=Z_{I P}(M M)$.

Proof. Let $\left(x^{*}, y^{*}, z^{*}\right)$ be an optimal solution to the LP relaxation of $(M M)$. Note that at optimality, we may assume

$$
y_{k}^{*}=\max _{m_{i}} \sum_{l \geq k} x_{i, i(l)}^{*}, \quad z_{k}^{*}=\max _{w_{j}} \sum_{l \geq k} x_{j(l), j}^{*} .
$$

We round $x^{*}$ to a stable marriage solution $\bar{x}$ using the rounding procedure in the proof of Theorem 1. Hence we have $E\left(\bar{x}_{i, j}\right)=x_{i, j}^{*}$. Let $y_{k}=\max _{m_{i}} \Sigma_{l \geq k} \bar{x}_{i, i(l)}$, and $z_{k}$ $=\max _{w_{j}} \Sigma_{l \geq k} \bar{x}_{j(l), j}$. Let $U$ be the random number generated in the rounding procedure.

$$
\begin{aligned}
E\left(y_{k}\right) & =P\left(\bigcup_{m_{i}}\left\{U \text { lies in the subinterval spanned by } x_{i, i(l)}^{*}, \text { for some } l \geq k\right\}\right) \\
& =\max _{m_{i}} \sum_{l \geq k} x_{i, i(l)}^{*}=y_{k}^{*} .
\end{aligned}
$$

Similarly, $E\left(z_{k}\right)=z_{k}^{*}$. Hence $Z_{\mathrm{LP}}(M M)=Z_{\mathrm{IP}}(M M)$.

To see that the above models the minimum-regret version of the stable marriage problem, we consider the case with $n$ men and $n$ women. Let $m=2 n$. If we set $f(k)=g(k)$ $=k^{m}$ for $k=1, \ldots, n$, and $c_{i, j}=0$ for all $i, j$, the above reduces to finding a stable marriage solution $x^{*}$, which is optimal for the objective function $\min \left(M(x)^{m}+W(x)^{m}\right)$. 
We claim that the solution $\bar{x}$ is also optimal for the objective function $\min \{\max (M(\bar{x})$, $W(\bar{x}))\}$. Suppose otherwise, then let $x^{\prime}$ be a stable marriage solution that is optimal for the latter objective function and

$$
\left\{\max \left(M\left(x^{\prime}\right), W\left(x^{\prime}\right)\right)\right\}+1 \leq\left\{\max \left(M\left(x^{*}\right), W\left(x^{*}\right)\right)\right\} .
$$

Since

$$
\begin{aligned}
\max \left(M\left(x^{*}\right), W\left(x^{*}\right)\right)^{m} & \leq\left(M\left(x^{*}\right)^{m}+W\left(x^{*}\right)^{m}\right) \\
& \leq\left(M\left(x^{\prime}\right)^{m}+W\left(x^{\prime}\right)^{m}\right) \leq 2 \max \left(M\left(x^{\prime}\right), W\left(x^{\prime}\right)\right)^{m},
\end{aligned}
$$

we have, by combining the two conditions that

$$
\left(1+\frac{1}{\max \left(M\left(x^{\prime}\right), W\left(x^{\prime}\right)\right)}\right)^{m} \leq 2 .
$$

Since $\max (M(\bar{x}), W(\bar{x})) \leq n$, we obtain $(1+1 / n)^{2 n} \leq 2$, which is a contradiction.

3. Fractional stable roommates polytope. Abeledo and Rothblum (1994) considered the following formulation for the roommates problem:

$$
\begin{gathered}
\sum_{j} x_{i, j}=1 \quad \forall i \\
x_{i, j}+\sum_{l: l<j i} x_{l, j}+\sum_{l: l<i j} x_{i, l} \leq 1 \quad \forall i, j . \\
x_{i, j} \geq 0 \quad \forall i, j .
\end{gathered}
$$

Constraints (14) must be valid, since otherwise, $j$ is matched to someone inferior to $i$ whereas $i$ is matched to someone inferior to $j$ and so $(i, j)$ is a blocking pair. We call constraints (14) the paired inequalities. Note that in the roommates case, the variable $x_{i, j}$ models the decision whether person $i$ is matched to person $j$.

By considering the dual of (FSM), and by the argument used in Lemma 1, Abeledo and Rothblum (1994) proved an analogous result for (FSM):

Lemma 2 (ABeledo AND Rothblum 1994). Let $x$ be a feasible solution in $(F S M)$. Then

$$
x_{i, j}>0 \quad \text { implies } x_{i, j}+\sum_{k: k<i} x_{i, k}+\sum_{k: k<j i} x_{k, j}=1
$$

REMARK. This formulation is slightly different from the one discussed by Abeledo and Rothblum (1994). Here, we insist that $\Sigma_{j} x_{i, j}$ be equal to 1 for every $i$, whereas they allowed $\Sigma_{j} x_{i, j}$ to be less than or equal to 1 . Curiously, this slight difference makes the above polytope infeasible in certain instances, whereas the polytope considered by Abeledo and Rothblum (1994) is always feasible. As an example, consider the following stable roommates problem, involving four people. The preference lists of the individuals are: 


$$
\begin{aligned}
& P(1): 2,3,4 \\
& P(2): 3,1,4 \\
& P(3): 1,2,4 \\
& P(4): 1,2,3
\end{aligned}
$$

We leave it to the reader to check that the above problem does not have a solution (no one can be matched to 4), and our formulation of this problem (FSM) does not have a solution either.

However, there are infeasible instances of the stable roommates problem for which $(F S M)$ is nonempty (see the 6 node example below), and so (FSM) cannot be used to address the feasibility question for the stable roommates problem. A natural way to improve the above formulation is to include the odd-set constraints from the matching polytope $\sum_{i, j \in S} x_{i, j} \leq\lfloor|S| / 2\rfloor$ for every set $S$ of odd cardinality. However, the following example shows that even the odd-set constraints are not strong enough:

Example (See Abeledo And Rothblum 1994). Consider the stable roommates problem with 6 nodes and the following preference lists:

$$
\begin{aligned}
& P(1): 2,3,5,6,4 \\
& P(2): 3,4,6,1,5 \\
& P(3): 4,5,1,2,6 \\
& P(4): 5,6,2,3,1 \\
& P(5): 6,1,3,4,2 \\
& P(6): 1,2,4,5,3
\end{aligned}
$$

It can be verified easily that vector $x$ with $x_{i, j}=\frac{1}{2}$ for $\{i, j\}$ in the set $\{\{1,2\},\{2,3\}$, $\{3,4\},\{4,5\},\{5,6\},\{6,1\}\}$ and $x_{i, j}=0$ otherwise is a feasible solution in (FSM) but there does not exist any stable roommates solution. Furthermore, the above $x$ is an extreme point of the polyhedron (FSM) and does not violate the odd-set constraints.

Using the proof technique of the previous section, we can show that the polyhedron $(F S M)$ is half integral. (This result has also been observed earlier by Abeledo and Rothblum 1994.)

\footnotetext{
Generate a uniform random variate $U$ in $\left(0, \frac{1}{2}\right]$. For each person $i$, arrange $x_{i, j}$ (for all $j$ ) to cover the interval $(0,1]$, in decreasing preference of $i$. Assign $i$ to $j$ if $U$ falls in the subinterval spanned by $x_{i, j}$. The assignment $X_{U}$ obtained is a union of disjoint cycles. $X_{U} / 2$ is then a half-integral solution in $(F S M)$.
}

The proof of the above statement follows from similar arguments as in the stable marriage polytope. Furthermore, the arrangement described above gives rise to an efficient procedure to express any fractional solution in (FSM) as a convex combination of halfintegral solutions in $(F S M)$. The main intuition behind these results is again the geometry (Figure 2) of the fractional solutions (cf. Lemma 2, we leave the details to the readers).

4. On a new class of valid inequalities. Let $x_{u, v}=1$ if $(u, v)$ is an edge in the matching, 0 otherwise. Consider distinct nodes $i, j, k$ such that $j$ prefers $k$ to $i$. For the matching to be stable, the following must be valid, 


\section{decreasing preference}

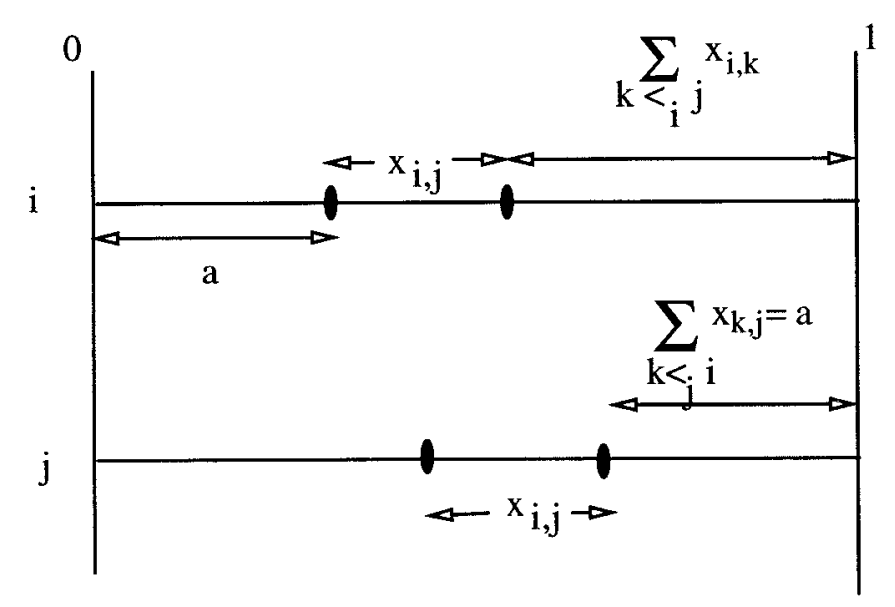

FIGURE 2. Geometry of a fractional solution in the stable roommates polytope.

$$
S(i, j, k) \equiv \frac{1}{2}\left(\sum_{l: l \leq j i} x_{l j}+\sum_{l: l \leq k j} x_{l k}\right) \leq \frac{1}{2}
$$

since the above is dominated by the paired inequalities (14) in (FSM).

The above inequality can be extended to an odd cycle version: Suppose $i_{0}, i_{1}, \ldots, i_{C}$ ( $C$ even) are such that $i_{k}$ prefers $i_{k+1}$ to $i_{k-1}$, where the indices are taken modulo $(C+1)$. Then by adding up the above inequality, we have

$$
\sum_{k=0}^{C} S\left(i_{k-1}, i_{k}, i_{k+1}\right) \leq \frac{C+1}{2} .
$$

Note that the nodes in the cycle need not be distinct. The coefficients of all variables appearing in the LHS are integral. Hence by rounding down the right-hand side, we have the following odd cyclic preference inequality:

$$
\sum_{k=0}^{C} S\left(i_{k-1}, i_{k}, i_{k+1}\right) \leq\left\lfloor\frac{C+1}{2}\right\rfloor .
$$

Thus we obtain an improved formulation:

$$
\begin{gathered}
\left(P_{S R}\right) \quad \sum_{j} x_{i, j}=1 \quad \forall i, \\
x_{i, j}+\sum_{l: l<j i} x_{l, j}+\sum_{l: l<i j} x_{i, l} \leq 1 \quad \forall i, j, \\
\sum_{k=0}^{C} S\left(i_{k-1}, i_{k}, i_{k+1}\right) \leq\left\lfloor\frac{C+1}{2}\right\rfloor \quad i_{k-1}<_{i_{k}} i_{k+1}, k=0, \ldots, C .
\end{gathered}
$$


Returning to the (FSM) polyhedron of the previous example ( 6 nodes), since $\Sigma_{l s_{3} 1} x_{l, 3}$ $=x_{2,3}=\frac{1}{2}, \Sigma_{l \leq 5} x_{l, 5}=x_{4,5}=\frac{1}{2}, \Sigma_{l \leq 5} x_{l, 1}=x_{6,1}=\frac{1}{2}$, this extreme point solution violates the odd cyclic preference inequality on nodes 1,3 and 5 . Note that the odd cyclic inequalities on $\{1,3,5\}$ and $\{2,4,6\}$ give rise to the following constraints,

$$
\sum_{e \in E(K)} x_{e} \leq 1, \quad \sum_{e \in E(H)} x_{e} \leq 1,
$$

where the graphs $K$ and $H$ are as shown in Figure 3.

The set $E(H) \cup E(K)$ contains all the edges in the graph and since $\Sigma_{e} x_{e}=3$ for any stable matching $x$, the above shows that this instance of the roommates problem is infeasible. (In our version of the stable roommates problem, we insist that all preference lists be complete, and that all participants be matched in any stable matching.)

The class of odd cyclic preference inequalities is exponential in number. We now describe a polynomial time separation routine for this class of inequalities, thus proving that optimizing a linear objective over $\left(P_{S R}\right)$ is solvable in polynomial time via the ellipsoid algorithm.

Separation routine. Note that separation over (FSM) (and hence (17)) is trivial since there are only $O\left(n^{2}\right)$ of these constraints. We define a new directed graph $G^{\prime}=\left(V^{\prime}, A^{\prime}\right)$ with

$$
\begin{aligned}
& V^{\prime}=\{(i, j): i \in V, j \in V\}, \\
& A^{\prime}=\left\{((i, j),(j, k)): i<_{j} k\right\} .
\end{aligned}
$$

For each $\operatorname{arc}((i, j),(j, k))$ in $A^{\prime}$, define a weight

$$
c(i, j, k)=1-\sum_{l: l \leq \leq_{j} i} x_{l j}-\sum_{l: l \leq k j} x_{l k} .
$$

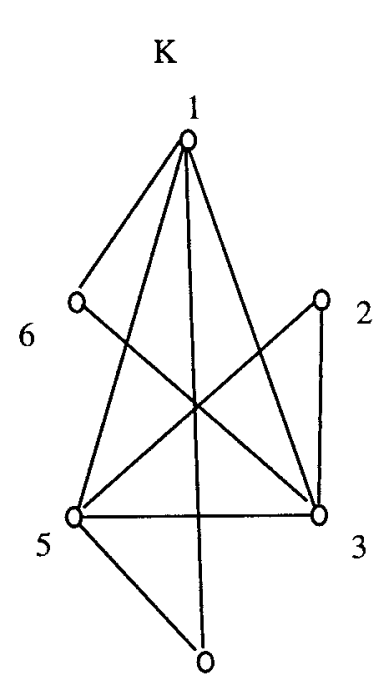

4
$\mathrm{H}$

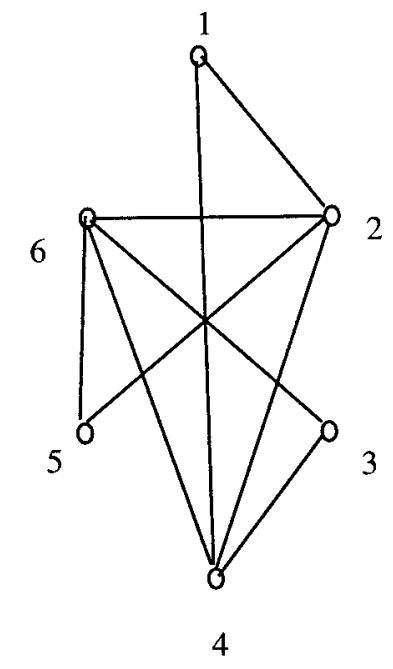

FIGURE 3. Certificate of infeasibility. 
Note that $c(i, j, k) \geq 0$ by (17). Furthermore, an odd directed cycle $e=\left(i_{0}, i_{1}, \ldots, i_{C}\right)$ (in that order) in $G^{\prime}$ gives rise to an odd cyclic preference inequality, with cost

$$
c(\Theta)=|\Theta|-2 \sum_{0 \leq k \leq C} \sum_{l: l \leq \leq_{i_{k}} i_{k-1}} x_{l, i_{k}} .
$$

Thus $c(\mathcal{C}) \geq 1$ if and only if $\sum_{0 \leq k \leq C} \sum_{l: l s_{i} i_{k-1}} x_{l, i_{k}} \leq(|\Theta|-1) / 2$, which is (18).

Note that finding a shortest odd cycle in the directed graph $G^{\prime}$ with a nonnegative weight function on the edges can be solved easily by solving $O\left(\left|V^{\prime}\right|\right)$ shortest directed path problems in an associated bipartite graph (see Grötschel, Lovász and Schrijver 1988).

5. Feasibility of the stable roommates problem. In this section, we prove the following result:

THEOREM 4. $\left(P_{S R}\right)$ is feasible if and only if the corresponding stable roommates problem is feasible.

Since one direction is easy, we prove the converse direction. As $\left(P_{S R}\right)$ is contained in $(F S M)$, we have:

LEMMA 3. If $x \in\left(P_{S R}\right)$, then

$$
x_{i, j}>0 \quad \text { implies } x_{i, j}+\sum_{l: l<i j} x_{l, i}+\sum_{l: l<j i} x_{l, j}=1 .
$$

We use the above lemma to derive a proof for the theorem using the following steps:

- For each person $i$, we arrange the $n$ subintervals $x_{i, j}$ as $j$ varies in decreasing preference of $i$. See also Figure 2. If $x_{i, k}$ is the subinterval that covers the point $\frac{1}{2}$, then we assign $k$ to $i$. In this way, the assignment gives rise to a union of disjoint cycles and a partial matching.

- Using the odd cyclic preference inequalities, we show that the length of each cycle arising this way must be even.

- We construct a stable matching from the union of even cycles and the partial matching.

Proof of Theorem 4. Suppose $x$ is a feasible solution in $\left(P_{S R}\right)$, we show how to construct a stable matching from $x$. Pictorially, if nonoverlapping intervals of length $x_{i, l}$ are arranged to cover the interval $(0,1]$ in decreasing preference of $i$, let $m(i)$ denote the node that corresponds to the interval that covers the point $\frac{1}{2}$. More formally $m(i)$ is the unique $j$ such that

$$
\sum_{k: k>i j} x_{i, k}<\frac{1}{2} \leq \sum_{k: k \geq_{i j}} x_{i, k}
$$

Furthermore, we denote $p(i)=j$ if $m(j)=i$. Note that if $x_{i, m(i)}$ corresponds to an interval $(a, b]$ such that $a<\frac{1}{2}<b$, then by Lemma 3, $m(m(i))=i$. Hence $i$ and $m(i)$ are matched to each other in our assignment. On the other hand, if $x_{i, m(i)}$ corresponds to an interval spanning ( $\left.a, \frac{1}{2}\right]$ for some $a$, then $m(m(i)) \neq i$. In that case, our assignment gives rise to a disjoint union of cycles and a partial matching. Let $e=\left(i_{0}, i_{1}, \ldots, i_{C}, i_{0}\right)$ be such a cycle in the assignment, with $m\left(i_{k}\right)=i_{k+1}$, and indices are taken with respect to modulo $(C+1)$.

Claim 1. $C$ is odd, i.e., $C$ is an even cycle.

Proof. Suppose $C$ is even. Since $m\left(i_{k-1}\right)=i_{k}$, the subinterval $x_{i_{k-1}, i_{k}}$ spans $\left(a_{k}, \frac{1}{2}\right]$ for some $a_{k}<\frac{1}{2}$, in the row corresponding to $i_{k-1}$ in our table. Hence in the row corresponding 
to $i_{k}$, the interval corresponding to $x_{i_{k}, i_{k-1}} \operatorname{covers}\left(\frac{1}{2}, 1-a_{k}\right.$ ] (by Lemma 3 ). So $\Sigma_{l \leq i_{k} i_{k-1}} x_{l, i_{k}}=\frac{1}{2}$. Summing for all $k=0$ to $C$, we have

$$
\sum_{k=0}^{C} \sum_{l \leq_{i} i_{k} k-1} x_{l, i_{k}} \geq \frac{C+1}{2}
$$

contradicting (18).

The above shows that the cycles arising from our assignment are even in length. We show next that we can extract two stable matchings from the edges of the union of disjoint even cycles and partial matching, denoted by $\mathscr{N}$.

Let an edge $(i, j)$ be an obstructing pair if $p(i)<_{i} j<_{i} m(i)$ and $p(j)<_{j} i<_{j} m(j)$. Note that for an obstructing pair $(i, j)$,

$$
\begin{aligned}
& \sum_{l: l \leq \leq_{i} i} x_{j, l} \geq \sum_{l: l \leq j p(j)} x_{j, l} \geq \frac{1}{2}, \\
& \sum_{l: l \leq j, j} x_{i, l} \geq \sum_{l: l \leq i p(i)} x_{i, l} \geq \frac{1}{2} .
\end{aligned}
$$

Note that the fractional value of $x_{i, j}$ is necessarily 0 if $\{i, j\}$ is an obstructing pair. By definition, if $(i, j)$ is an obstructing pair, then the node $i$ (and also $j$ ) must be in the vertex set of some even cycle $C$ in $\mathscr{X}$.

Claim 2. Let $M$ be any matching extracted from $\mathscr{X}$. Then $(i, j)$ is a blocking pair for $M$ only if $(i, j)$ is an obstructing pair. Furthermore, no blocking pair exists for two nodes on a common cycle in $\mathscr{X}$.

Note that the definition of obstructing pair is independent of the matching extracted from $\mathscr{X}$.

Proof. Let $(i, j)$ be a blocking pair for the matching $M$. We prove the statement by considering several cases.

Case 1. Suppose the matching $M$ contains $(i, m(i)),(j, m(j))$. By the definition of a blocking pair, $j>{ }_{i} m(i)$ (the interval $x_{i, j}$ is on the left of $x_{i, m(i)}$ for node $i$, i.e., strictly in $\left.\left(0, \frac{1}{2}\right)\right)$, and by Lemma 3 , for the arrangement by $j$, the subinterval spanned by $x_{i, j}$ lies strictly in $\left(\frac{1}{2}, 1\right)$. Hence $m(j)>_{j} i$ (interval $x_{j, m(j)}$ on the left of $x_{i, j}$ for node $j$ ). This contradicts the fact that $(i, j)$ is a blocking pair.

Case 2. Suppose the matching contains $(i, p(i)),(j, p(j))$. Since $j>_{i} p(i)$, and if $j \geq_{i} m(i)$, we have $i \leq_{j} p(j)$ by Lemma 3, contradicting the fact that $(i, j)$ is a blocking pair. Hence we must have $m(i)>_{i} j>_{i} p(i)$. By symmetry, we also have $m(j)>_{j} i$ $>_{j} p(j)$, i.e., $(i, j)$ is an obstructing pair.

Case 3. Suppose the matching contains either $(i, m(i)),(j, p(j))$ or $(i, p(i)),(j$, $m(j))$. Now $j>_{i} m(i)\left(\right.$ or $\left.i>_{j} m(j)\right)$ only if $i<_{j} p(j)\left(\right.$ resp. $\left.j<_{i} p(i)\right)$. Hence $(i, j)$ cannot be a blocking pair.

The above shows that $(i, j)$ is a blocking pair of $M$ (and hence an obstructing pair) only if $(i, p(i)),(j, p(j))$ belongs to the matching $M$. Suppose now $(i, j)$ is a blocking pair. If $i, j$ are nodes on a common cycle $C$ in $\mathscr{X}$, say $C=\left(i_{0}, i_{1}, \ldots, i_{C}\right)$, with $C$ odd, then by the preceding argument, both $i, j$ are matched by $M$ to their respective predecessors in the cycle. This implies that they are an even distance apart, and therefore both cycles formed by the addition of the chord $(i, j)$ are odd. That is, both cycles $\left(i_{0}, \ldots, p(i), i\right.$, 
$\left.j, m(j), \ldots, i_{C}\right)$ and $(j, i, m(i), \ldots, p(j))$ are odd. Consider the former cycle. Note that by our assignment, $\Sigma_{l \leq_{i, p}\left(i_{k}\right)} x_{l, i_{k}}=\frac{1}{2}$. Furthermore, $\Sigma_{l \leq_{j} i} x_{l, j} \geq \Sigma_{l \leq j p(j)} x_{l, j}=\frac{1}{2}$. Summing over all the nodes in the odd cycle, we obtain an odd cyclic preference inequality that violates (18).

The above argument yields the last statement in the claim.

Let $G^{*}=\left(V, E^{*}\right)$ denote the graph defined on $V$ with edge set consisting of $\mathscr{X}$ and all obstructing pairs, excluding those obstructing pairs occurring on nodes of common cycle in $\mathscr{X}$. Note that the added set of edges contains all the blocking pairs in any matching extracted from $\mathscr{X}$.

Claim 3. $G^{*}$ is bipartite.

Proof. Suppose $G^{*}$ contains an odd cycle $\mathscr{D}$, consisting of a union of copies of paths from $\mathscr{X}$ and obstructing edges. Fix an orientation for $\mathscr{D}$. Denote the directed odd cycle by $\overline{\mathcal{D}}$. We use the following modification to obtain a new directed cycle $\overline{\mathcal{D}}^{\prime}$ from $\bar{\Phi}$ :

- For arc $(i, j)$ in $\bar{\Phi}$ which corresponds to an obstructing pair, insert an even directed cycle $C^{\prime}=(j, m(j), \ldots, p(j))$ in $\bar{D}$ at the node $j . C^{\prime}$ is the even cycle (oriented) in $M$ containing the node $j$.

- For a path in $\mathscr{D}$, say along the cycle $e$ in $\mathscr{X}$, if the orientation of the arcs in $\bar{D}$ are from $j$ to $m(j)$, do nothing. If the orientation is from $j$ to $p(j)$, replace the path by its complement in $C$, with each node oriented from $j$ to $m(j)$.

In this way, we maintain the parity of the number of nodes in the directed cycle. Furthermore, all the arcs that correspond to edges in $\mathscr{X}$ are of the type $(j, m(j))$, i.e., oriented from $j$ to $m(j)$. Every arc that corresponds to an obstructing pair is isolated in the new directed cycle $\overline{\mathscr{D}}^{\prime}$. In this way, for every triplet $(i, j, k)$ of consecutive nodes in $\bar{D}^{\prime}, i<{ }_{j} k$ and $S(i, j, k) \geq \frac{1}{2}$. This gives rise to an odd cyclic preference inequality, which violates (18).

Since $G^{*}$ is bipartite, let $A$, and $B$ be the two partite sets of $G^{*}$. This splits the nodes of $\mathscr{X}$ into two parts each of equal size. Since blocking pair $(i, j)$ exists only if ( $i$, $p(i))$ and $(j, p(j))$ belong in the matching, and since there are no obstructing pairs (hence no blocking pair) for any two nodes both in $A$ or in $B$, the assignments $\{(a$, $m(a)): a \in A\}$ and $\{(a, p(a)): a \in A\}$ are two stable matchings for the roommates problem.

This concludes the proof for the theorem.

Finally, we note the following interesting structural properties of the stable roommates solutions (the proofs are straightforward and are left as exercises for the reader).

THEOREM 5. Let $X_{1}, X_{2}, \ldots, X_{l}$ be l distinct stable roommates solutions, and assume $l$ is odd. Each person has l possible roommates under these matchings. To each person, assign the person whose rank is $(l+1) / 2$ among the $l$ (possibly nondistinct) roommates. This assignment gives rise to another stable roommates solution.

THEOREM 6. Let $X_{1}, X_{2}, \ldots, X_{l}$ be l distinct stable roommates solutions, and assume $l$ is even. Each person has l possible roommates under these matchings. Then there is a stable roommates solution in which each person is assigned to a person whose rank is $1 / 2$ or $l / 2+1$ among the l possible roommates.

5.1. Optimal stable roommates problem. In the rest of this paper, we address the effectiveness of this LP approach to the minimum cost version of the stable roommates problem. Suppose the cost function is of the type $d_{i, j}=c(i, j)+c(j, i)$ for each edge $(i, j)$, where the cost function $c(\cdot, \cdot)$ is assumed to be nonnegative. We show that the stable roommates solution constructed from the optimal solution to $\left(P_{S R}\right)$ is within a factor 
of 2 from the optimal LP solution, if the cost function $c$ satisfies the following condition: For each $i$, with preference list $i_{1}<_{i} \cdots<_{i} i_{n}$, there is a node $i_{k}$ such that

$$
c\left(i, i_{1}\right) \geq \cdots \geq c\left(i, i_{k-1}\right) \geq c\left(i, i_{k}\right) \leq c\left(i, i_{k+1}\right) \leq \cdots \leq c\left(i, i_{n}\right) .
$$

We say the cost function $c$ satisfies the $U$-shape condition in this case. Let $x *$ denote an optimal solution to

$$
Z_{\mathrm{LP}}=\min \left\{\sum_{\{i, j\} \in E(G)} d_{i, j} x_{i, j}: x \in\left(P_{S R}\right)\right\}
$$

Let $M$ be a matching constructed from $x^{*}$, using the procedure described in the proof of Theorem 4. Let $d(M)$ denote the weight of the matching $M$ under the function $d$.

THEOREM 7. $d(M) \leq 2 Z_{L P}$.

Proof. Let $(i, j)$ be an edge in $M$, and let $i_{k}$ denote the index with the least cost among all $c(i, k)$ as $k$ varies.

Suppose $m(i)=j$. In the arrangement according to $i$, the interval corresponding to $x_{i, j}^{*}$ spans $(a, b]$ where $a<\frac{1}{2}$ and $b \geq \frac{1}{2}$. Hence for the arrangement according to node $j, x_{i, j}^{*}$ spans $(1-b, 1-a]$ (by Lemma 3 ). If $i_{k} \leq_{i} j$, then since $c$ satisfies the $U$-shape condition,

$$
c(i, j) \times \frac{1}{2} \leq c(i, j)\left(\left(\frac{1}{2}-a\right)+\sum_{l: l>i j} x_{i, l}^{*}\right) \leq c(i, j) x_{i, j}^{*}+\sum_{l: l>i j} c(i, l) x_{i, l}^{*} .
$$

On the other hand, if $i_{k}>_{i} j$, then

$$
c(i, j) \times \frac{1}{2} \leq c(i, j)\left(\left(b-\frac{1}{2}\right)+\sum_{l: l<j} x_{i, l}^{*}\right) \leq c(i, j) x_{i, j}^{*}+\sum_{l: l<j} c(i, l) x_{i, l}^{*} .
$$

Hence $c(i, j) \leq 2 \Sigma_{l} c(i, l) x_{i, l}^{*}$.

Similarly, $c(j, i) \leq 2 \Sigma_{l} c(j, l) x_{j, l}^{*}$. So we have $d(M) \leq 2 Z_{\mathrm{LP}}$ and the result follows.

6. Concluding remarks. In this paper, we study stable matching problems by exploiting an interesting structural property of the fractional solutions. This property also allows us to express fractional solutions in the stable marriage polytope as convex combinations of integral solutions, in a completely geometric way. For the roommates problem, we propose a stronger formulation that can be used to address the question of whether a stable roommates solution exists. This complements the purely combinatorial approach used by Irving (1985) in his solution to the above problem. From a methodological perspective, our rounding approach is an extension of the idea of randomized rounding originating from Raghavan and Thompson (1987). By suitably exploiting the structural properties of the fractional solution, we show how a stable matching can be constructed from the fractional solution. The formulation studied here can also be used naturally to study the optimal stable roommates problem. A 2-approximation algorithm can be obtained from our approach if the cost function satisfies a certain $U$-shape condition. In addition, it is conceivable that by adding more classes of valid inequalities, the approximation guarantee obtained here can be improved further. 
7. Acknowledgments. We thank Professor Dimitris Bertsimas, Professor Rakesh Vohra, Viji Srinivasan and the referees for their extensive comments and for pointing out errors and omissions in an earlier draft of this paper. We are especially grateful to one of the referees for filling a gap in the proofs of Claim 2 and Theorem 7.

\section{References}

Abeledo, H. G., Y. Blum. 1996. Stable matchings and linear programming. Linear Algebra Appl. 245321 -333.

- , U. G. Rothblum. 1996. Canonical monotone decompositions of fractional stable matchings. Internat. J. Game Theory 25 161-176.

, U. G. Rothblum. 1994. Stable matching and linear inequalities. Discrete Appl. Math. 54 1-27.

Feder, T. 1992. A new fixed point approach for stable networks and stable marriages. J. Comput. System Sci. 45 233-284.

Gale, D., L. S. Shapley. 1962. College admissions and the stability of marriage. Amer. Math. Monthly 69 915.

Grötschel, M., L. Lovász, A. Schrijver. 1988. Geometric Algorithms and Combinatorial Optimization, SpringerVerlag, Berlin.

Gusfield, D., R. W. Irving. 1989. The Stable Marriage Problem: Structure and Algorithms, MIT Press, Massachusetts.

Irving, R. W. 1985. An efficient algorithm for the stable roommates problem. J. Algorithms 6 577-595.

, P. Leather, D. Gusfield. 1987. An efficient algorithm for the optimal stable marriage problem. J. ACM $34532-543$.

Karp, R. M., C. H. Papadimitriou. 1982. On linear characterizations of combinatorial optimization problems. SIAM J. Comput. 11 620-632.

Knuth, D. E. 1976. Marriages Stables, Les Presses de l'Université de Montreal, Montreal.

Raghavan, P., C. Thompson. 1987. Randomized rounding: A technique for provably good algorithms and algorithmic proofs. Combinatorica 7 365-374.

Roth, A. E. 1984. The evolution of the labor market for medical interns and residents: A case study in game theory. J. Political Economy 92 991-1016.

— U. G. Rothblum, J. H. Vande Vate. 1993. Stable matching, optimal assignments and linear programming. Math. Oper. Res. 18 808-828.

, M. Sotomayor. 1991. Two-Sided Matching: A Study in Game-Theoretic Modeling and Analysis, Cambridge University Press, Cambridge.

Rothblum, U. G. 1992. Characterization of stable matchings as extreme points of a polytope. Math. Programming $5457-67$.

Subramanian, A. 1994. A new approach to stable matching problems. SIAM J. Comput. 23 671-700.

Teo, C. P., J. Sethuraman. 1997. LP based approach to optimal stable matchings. Proceedings of the Eighth Annual ACM-SIAM Symposium on Discrete Algorithms (New Orleans, LA) 710-719.

Vande Vate, J. H. 1989. Linear programming brings marital bliss. Oper. Res. Lett. 8 147-153.

Chung-Piaw Teo: Department of Decision Sciences, Faculty of Business Administration, National University of Singapore; e-mail: fbateocp@nus.edu.sg

Jay Sethuraman: Operations Research Center, MIT, Cambridge, Massachusetts 02139; e-mail: jayc@ mit.edu 